\title{
Automated Finite Element Meshing of the Lumbar Spine: \\ Verification and Validation with 18 Specimen-Specific Models
}

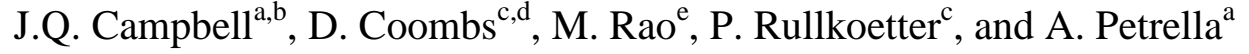 \\ ${ }^{\text {a }}$ Department of Mechanical Engineering, Colorado School of Mines, 1500 Illinois St., Golden, Colorado, USA \\ ${ }^{\mathrm{b}}$ Vector Scientific Inc., Colorado, USA \\ ${ }^{\mathrm{c}}$ Department of Mechanical and Materials Engineering, University of Denver, Colorado, USA \\ ${ }^{\mathrm{d}}$ DePuy Synthes Trauma, Pennsylvania, USA \\ ${ }^{\mathrm{e}}$ BD Medical - Medication \& Procedural Solutions, New Jersey, USA
}

\begin{abstract}
The purpose of this study was to seek broad verification and validation of human lumbar spine finite element models created using a previously published automated algorithm. The automated algorithm takes segmented CT scans of lumbar vertebrae, automatically identifies important landmarks and contact surfaces, and creates a finite element model. Mesh convergence was evaluated by examining changes in key output variables in response to mesh density. Semi-direct validation was performed by comparing experimental results for a single specimen to the automated finite element model results for that specimen with calibrated material properties from a prior study. Indirect validation was based on a comparison of results from automated finite element models of 18 individual specimens, all using one set of generalized material properties, to a range of data from the literature. A total of 216 simulations were run and compared to 186 experimental data ranges in all six primary bending modes up to $7.8 \mathrm{Nm}$ with follower loads up to $1000 \mathrm{~N}$. Mesh convergence results showed less than a $5 \%$ difference in key variables when the original mesh density was doubled. The semi-direct validation results showed that the automated method produced results comparable to manual finite element modeling methods. The indirect validation results showed a wide range of outcomes due to variations in the geometry alone. The studies showed that the automated models can be used to reliably evaluate lumbar spine biomechanics, specifically within our intended context of use: in pure bending modes, under relatively low non-injurious simulated in vivo loads, to predict torque rotation response, disc pressures, and facet forces.
\end{abstract}




\section{Introduction}

Finite element (FE) modeling of the lumbar spine holds promise for pre-clinical evaluation of new devices, but subject-specific modeling and simulation remain challenging due to the uncertainty of material properties and boundary conditions (Dreischarf et al., 2014).

Alternatively, population-based modeling has the potential to offer information on device efficacy across a broad range of virtual patients, and parameter uncertainty can be accommodated with well-established probabilistic methods. Modeling a large number of subjects to represent a target population has been impractical, however, due to the complexity of spinal geometry and the time consuming process of model creation. A new method for automatic generation of FE meshes of the lumbar spine addresses this limitation (Campbell and Petrella, 2015), but it has not been formally evaluated, which was the focus of the present study. Verification and validation $(\mathrm{V} \& \mathrm{~V})$ are the primary methods for evaluating the reliability and accuracy of an FE model within its context of use. Several papers have been written in the last decade highlighting the importance of $\mathrm{V} \& \mathrm{~V}$ and providing guidelines for these methods in the field of biomechanics (Anderson et al., 2007; Erdemir et al., 2012; Henninger et al., 2010; Viceconti et al., 2005). These authors have also helped to reinforce a standard vocabulary around $\mathrm{V} \& \mathrm{~V}$. Namely, verification is the process of confirming the computational methods solve the governing equations correctly and accurately. Validation involves evaluating how accurately the model simulates the real physical system of interest.

Jones and Wilcox presented a specific framework for $\mathrm{V} \& \mathrm{~V}$ in the spine (Jones and Wilcox, 2008), and highlighted the importance of presenting verification results in the form of FE mesh convergence. However, relatively few studies based on segment FE models of the spine have reported detailed investigations of mesh convergence. Some studies of the lumbar spine have 
1 evaluated the FE mesh qualitatively (Eberlein et al., 2004, 2001; Tsouknidas et al., 2013), but the

2 study by Ayturk and Puttlitz is one of the few to report a detailed quantitative mesh convergence

3 analysis (Ayturk and Puttlitz, 2011). They evaluated a multi-segment lumbar spine model using

4 multiple bending modes and outcome metrics with a convergence criterion of $5 \%$.

5 Most validation studies for segment FE models of the spine have also used indirect validation

6 (Jones and Wilcox, 2008). Indirect validation for a spine FE model usually involves comparing

$7 \quad$ FE output metrics to a range of experimental data from multiple specimens to demonstrate that

8 the FE predictions fall within a reasonable experimental spread. This is in contrast to direct

9 validation which typically involves developing an FE model of a specific specimen and comparing FE results to experimental data from the same specimen. Direct validation of segment

11 models of the spine presents significant challenges as both the geometry and material properties

12 of a specific specimen need to be known to develop an accurate subject-specific FE model.

13 The purpose of the current study was to seek broad verification and validation of lumbar

14 spine FE models created using a previously published automated algorithm (Campbell and

15 Petrella, 2015). We defined our context of use as: prediction of torque-rotation response, disc

16 pressure, and facet force in flexion/extension, axial rotation, and lateral bending, under typical in

17 vivo simulated loads. A detailed, quantitative mesh convergence study was conducted to verify

18 appropriate mesh density created by the automated method. Semi-direct validation was

19 performed by using calibrated material properties and precise boundary conditions for a single

20 specimen from a prior experimental study (Coombs et al., 2013; Rao, 2012). A thorough indirect

21 validation was also performed for 18 full lumbar FE models using generalized material

22 properties and a range of experimental outcomes reported in the literature. 


\section{2. Materials and methods}

\section{2.1. FE Model Details}

3 Briefly, the automated algorithm used to create the FE models in this study accepts STL

4 geometry of lumbar vertebrae as input and identifies 1,306 landmarks characterizing the key

5 biomechanical features of each bone, such as endplate contours, ligament attachment points, and

6 facet contact surfaces. Landmark data are then used as the basis for automated fitting of a pre-

7 existing template FE mesh (Coombs et al., 2013; Rao, 2012) to the subject-specific bone

8 geometry. All models are oriented with the vertical axis of L4 aligned with gravity. Lastly, soft

9 tissues are also added to create a complete Abaqus (Simulia, Johnston, RI, USA) FE model of

10 the specimen. The automated algorithm runs in Matlab (MathWorks, Natick, MA) and requires

11 approximately 90 minutes to complete for a typical multi-segmental lumbar specimen (L1-L5).

12 Additional details of the automated methods may be found in (Campbell and Petrella, 2015).

13 Three specific workflows were used to evaluate the FE models created by the automated

14 algorithm (Fig. 1). Specimen geometry, material properties, and the FE solver were chosen

15 deliberately for efficiency and for direct comparison to previous work where appropriate. A

16 single representative L4-L5 functional spinal unit (FSU) was used for mesh convergence (Fig.

17 1a). Semi-direct validation was performed using the automated method with bone geometry and

18 calibrated material properties (Fig. 1b), both of which were obtained from a prior study (Rao,

19 2012). And, indirect validation was based on a comparison of results from automated FE models

20 of 18 individual specimens to a range of data from the literature (Fig. 1c). For mesh convergence

21 and indirect validation, a set of generalized material properties was synthesized from the

22 literature (Table 1). 
The bones and endplates were represented by 7470 elements total for each vertebra and were modeled as rigid, similar to several previous studies (Cegoñino et al., 2014; Coombs et al., 2013; Dreischarf et al., 2014; Little et al., 2007; Moramarco et al., 2010; Rao, 2012). Since the bone elements were rigid, their only function in the model was for visualization of the bone surface and confirmation of correct soft tissue attachment. Seven relevant spinal ligaments were represented using sets of nonlinear tension-only connector elements as described in Campbell and Petrella (2015). The nonlinear ligament properties were based on exponential fits from the literature (Ayturk and Puttlitz, 2011; Nolte et al., 1990; Rohlmann et al., 2006).

The annulus fibrosis was modeled using the Holzapfel-Gasser-Ogden material formulation in Abaqus with 3108 linear hexagonal elements per disc. This material model allows a continuous hexagonal mesh to represent a NeoHookean ground matrix with fiber reinforcement. The continuous mesh is of particular importance using the automated methods because the shape and size of the disc elements change depending on the specimen geometry. The Holzapfel-GasserOgden material model allows fiber angles to be defined numerically so that orientation of the fiber network is maintained irrespective of morphological changes in the continuum. Hybrid elements were used for the annulus fibrosis to allow incompressible behavior without shear locking (Eberlein et al., 2004, 2001). The annulus fibrosis matrix properties were derived from Eberlein et al. (2004, 2001). The annulus fibrosis properties were divided into anterior, lateral, and posterior regions (Rao, 2012). A different fiber stiffness was defined in each region (Eberlein et al., 2004, 2001; Malandrino et al., 2013) and the fiber angle was based on the average angle for each region in a regression model (Holzapfel et al., 2005). The generalized material parameters are summarized in Table 1. 
The nucleus pulposus was modeled as a fluid cavity in Abaqus using 2154 quadrilateral

2 reduced integration surface elements (SFM3D4R) per disc to define the nucleus boundary. The fluid cavity definition in Abaqus represented the nucleus pulposus with homogeneous fluid pressure. The cavity was defined with the bulk modulus of water based on the template model (Rao, 2012) and consistent with other fluid cavity models from the literature (Charles et al., 2013; Niemeyer et al., 2012). The facet cartilage was modeled using rigid hexagonal elements with 864 elements for each section of superior cartilage and 336 elements for each section of inferior cartilage. Rigid contact surfaces were created based on the geometry of each set of rigid cartilage elements. The facet contacts were defined using frictionless softened contact with a linear pressure-overclosure relationship to define interaction of the sets of rigid cartilage surfaces. The initial gap between the cartilage surfaces was defined relative to the subchondral bone as identified on the CT for each specimen (Campbell and Petrella, 2015). The ligament endpoints, superior, and inferior surfaces of the discs, and interior surface of the cartilage elements were all rigidly fixed to the bones where they attached.

\subsection{Mesh Convergence Study}

The mesh convergence study was performed to confirm adequate mesh density such that the key variables in our context of use were not influenced significantly by mesh density. The mesh was considered converged if the key output variables changed less than $5 \%$ when the mesh density was doubled. A single L4-L5 FSU from one arbitrarily chosen auto-generated FE model was used. Pure moment loads up to $10 \mathrm{Nm}$ were applied to the model in each of the six primary bending modes (flexion, extension, left/right axial rotation, and left/right lateral bending). Rotation, disc pressure, and facet contact force were used as output variables. 
1

2

3

4

5

6

7

8

10

11

12

\subsection{Semi-direct Validation Study}

The semi-direct validation study was conducted to evaluate the FE automation method by comparing simulation results to experimental measurements and results from a manually created

FE model. The semi-direct validation comparison specifically assessed the ability of the automation algorithm to create a valid, properly configured subject-specific FE model. Material properties were obtained from a prior study (Rao, 2012) that calibrated an FE model to experimental tests on a lumbar spine with progressive sectioning of the ligaments. The experimental study reported torque rotation curves for each level of the L1-S1 lumbar spine in all six primary bending modes for both the experiment and calibrated model. In the current study, CT data for the same specimen was used as input to the automated FE model generation method (Campbell and Petrella, 2015). The published method was designed for L1-L5 models, so the method was adapted for use with S1 for the semi-direct validation study only. The material properties and boundary conditions were identical to the Rao model, and the simulations were run in Abaqus Explicit to replicate their methods. The sacrum was fixed in space and pure torque was applied to L1. A $450 \mathrm{~N}$ follower load was applied during flexion and extension, consistent with the experiments. An example of the model in each of the six bending modes is shown in Figure 2. The torque-rotation results of the current study were compared to the experimental results and to Rao's FE results. RMS (root mean squared) error was calculated for each bending direction. RMS error was reported for the full L1-S1 rotation and an average RMS error was reported for the FSUs by averaging the RMS error for rotation of each of the five FSUs.

\subsection{Indirect Validation Study}


The indirect validation study was conducted to broadly evaluate the biomechanics of models

2

3 (i.e., several specimens) to experimental data from the literature. This evaluation also provided

4 an opportunity to compare outcomes from the auto-generated models to other lumbar spine FE

5 models in the literature.

6 Eighteen full lumbar FE models (L1-L5, see Fig. 3), were created using the automation

7 algorithm. Inputs to the automated algorithm were STL models of bone geometry manually

8 segmented from anonymized CT scans of varying resolution and unknown demographic

9 background. The same set of generalized material properties described above (Table 1) was used

10 for all 18 models. The outcome metrics of interest for the indirect validation were rotation, disc

11 pressure, and facet force. A set of 12 simulations was run for each of the models consistent with

12 other recently published validation studies (Ayturk and Puttlitz, 2011; Dreischarf et al., 2014).

13 Six simulations were conducted to evaluate the six pure bending modes to in vitro experimental

14 data up to $7.5 \mathrm{Nm}$. Six additional simulations were conducted to compare the model to in vivo

15 experimental data. The simulated in vivo loading conditions were based on Dreischarf et al.

16 (2014) and included one pure compression run and bending runs with follower loads in all of the

17 six primary modes except flexion (Dreischarf et al., 2014). Flexion was excluded because

18 Dreischarf et al. concluded that their originally proposed torque was not consistent with in vivo

19 loading.

20 The results of the indirect validation study were compared to a range of available data in the

21 literature that contained the variables of interest and replicated the simulated loading conditions.

22 Both in vivo and in vitro data were used for torque-rotation and disc pressure. Only in vitro data 
1 were used for facet force. In total 186 experimental data ranges were used for comparison to

2 each of the 18 FE models.

\section{3. Results}

4 3.1. Mesh Convergence Results

5 The mesh convergence results showed the percent change between the original mesh and

6 dense mesh for each of the key variables of interest. There was less than a 5\% difference in each

7 of the key variables between the two mesh densities, and most variables had less than a $2 \%$

8 change (Figure 4). The original mesh, therefore, was found to produce well-converged results

9 with element edge lengths of approximately $1-1.5 \mathrm{~mm}$ throughout the disc and facet contact 10 interfaces.

\section{$11 \quad 3.2 . \quad$ Semi-direct Validation Results}

12 The semi-direct validation involved a comparison of the automated FE model from the 13 current study to the experimental results and the FE results from Rao (2012). The torque rotation 14 curves are presented for each of the three datasets in all six bending modes (Figure 5). The 15 rotations were also evaluated at each of the five FSU levels. The RMS error for the automated 16 FE model for L1-S1 rotation ranged from 0.6-1.1 degrees versus Rao 2012 and 1.7-3.3 degrees

17 versus the experiments (Table 2). The RMS error for the FSUs averaged over all five levels 18 ranged from 0.1-0.2 degrees versus Rao 2012 and 0.3-0.6 degrees versus the experiments (Table $192)$.

\section{3.3. Indirect Validation Results}


The indirect validation study includes results from 216 simulations; 12 runs for each of the

2

3

4

18 automatically generated L1-L5 FE models. A total of eleven of the 2016 simulations failed to converge at some point in the run. The results of the FE simulations have been compared to several experimental data sets. The torque-rotation results for in vitro flexion, extension, and lateral bending show a wide range of responses due to geometrical variation alone (Figure 6).

Many of the specimen geometries produced results that fell within the bounds of the experiments (Guan et al., 2007), but several geometries produced results that were stiffer than the experiments (especially in lateral bending) or softer than the experiments (primarily in extension). The experimental data were reported every $0.5 \mathrm{Nm}$ from $0-4 \mathrm{Nm}$ (128 data ranges). The remaining 53 experimental data ranges and corresponding simulations include data on rotation, facet force, and disc pressure (Figure 7). The simulation results for in vitro loading showed a wide variation in all of the results due to geometric variation alone; often similar to the ranges reported in the experiments. However, the simulated in vivo loading produced a much narrower range than the experiments.

\section{Discussion}

The current study involved a mesh convergence study, a semi-direct validation of one automatically generated subject-specific FE model, and an indirect validation of 18 automatically generated FE models with subject-specific geometries. The mesh convergence study showed that the original mesh density was sufficient based on differences under $5 \%$ (most under $2 \%$ ) in flexion, extension, lateral bending, and axial rotation for key metrics of rotation, disc pressure, and facet force. A limitation of the mesh convergence is that only one FSU (L4-L5) from one subject was used. While this one FSU represented the mesh topology used in all other FSUs and specimens, changes in geometry could produce different mesh convergence results in other areas. 
1 Ideally mesh convergence would be performed on every model produced, but that is usually not

2 practical. Choosing a representative model for convergence is consistent with the methods of

3 other automated modeling techniques in the literature (Bischoff et al., 2014; Galloway et al.,

4 2013).

5 The semi-direct validation results show that the automated method was able to produce

6 results comparable to manual FE modeling methods. With prior calibrated material properties the

7 model produced torque-rotation curves in good agreement with experimental measurements.

8 Results were in good agreement at both the full lumbar level (L1-S1) and for individual FSUs,

9 providing a modest level of hierarchical validation - that is, we observed good agreement with

10 experiment for both constituent parts (FSUs) as well as the full lumbar model. Improvements for

11 semi-direct validation of subject-specific models could be made with modifications to the

12 automated FE algorithm to better treat soft tissues. The facet surfaces were based on the

13 geometry of the subchondral bone. Linked CT and MRI datasets could be used to include

14 subject-specific facet cartilage thicknesses and to include subject-specific geometry and material

15 properties for the intervertebral discs.

16 The lumbar spine FE models used in this study were created considering our context of use.

17 A relatively simple and efficient FE model setup was employed using rigid bones, rigid

18 endplates, and ligaments meshed with 1-D structural elements. These choices improved

19 simulation run times which was important considering the large number of models and

20 simulations completed. While these models are certainly not appropriate for all applications,

21 similar FE models have been published and validated for situations with relatively low loads

22 (Cegoñino et al., 2014; Dreischarf et al., 2014; Little et al., 2007; Moramarco et al., 2010; Rao, 
1 2012). Validation for higher loads, as studied by others (Shirazi-Adl et al., 2006), or other key

2 variables of interest would likely need to employ more complex modeling methods.

3 The goal of our indirect validation was to evaluate a set of subject-specific model geometries

4 all using the same general material properties. In the true human population material properties

5 in the lumbar spine vary considerably and affect biomechanics (Lee and Teo, 2005). Therefore, it

6 makes sense that our models do not match the full range of results from the general population

7 because we only included geometric variation in 18 subjects and no material property variation.

8 Similarly, it is reasonable that not all of our results remain within the bounds of the experimental

9 data because often there were fewer subjects in the experimental tests than there were in the

10 simulations. The relatively wide distribution of our results, all using the same material

11 properties, is an indication that the 18 specimens modeled exhibit a relatively wide range of

12 geometric variation, and this variation was responsible for substantial variability in the

13 biomechanical outcome measures.

14 Overall we found that our models compared reasonably well to the experimental data. The

15 results suggest that our general material properties may be stiffer than average for flexion and

16 lateral bending and may be softer than average for extension. The response of the models

17 appeared close to the average for axial rotation. However, our goal was not to determine average

18 material properties for the population but to evaluate generic FE models with different

19 geometries. Facet force results showed similar trends to the experimental data in different

20 bending modes. Facet forces were generally higher in the simulations than the experiments for

21 the modes of primary interest: extension and axial rotation. These differences may be due to the

22 FE models and/or reliability of the experimental data. While these experiments are currently the

23 best available data for facet force, they have primarily been aimed at methods to measure facet 
1 force, not establishing a range of facet forces in the population. They also have significant

2 potential limitations in terms of accuracy, calibration, and in some cases compromise of the facet

3 joint capsule.

4 Disc pressures in the simulations in general compared favorably to the experimental data for 5 compression and bending modes at multiple spinal levels. The indirect validation results for the

6 automated FE models are comparable to other full lumbar spine FE models in the literature

7 (Ayturk and Puttlitz, 2011; Dreischarf et al., 2014). Dreischarf et al. (2014) published a paper

8 similar to the current study by comparing eight FE models to similar experimental data.

9 However, all eight models were constructed by different institutions, with different methods, 10 using different FE solvers, and different material properties. To our knowledge this is the first 11 study to create and evaluate many full lumbar FE models of different specimens using the same 12 modeling methods.

13 We attempted to validate the models against a broad and thorough dataset. We feel that these 14 results indicate the FE models and automated methods can reasonably be used within the range 15 of validation tests performed (i.e. non-coupled bending modes with and without follower loads) 16 for the metrics tested (i.e. rotation, facet force, and disc pressure). Facet force should be used 17 with caution because the forces predicted were generally higher than the experiments, but the 18 trends appeared to be reliable. Therefore, the model can be considered validated for studies that 19 look at how variations in geometry, material properties, or implants affect trends in the validated 20 output variables within the loads studied.

21 Additional direct or semi-direct validation would be necessary to determine if the models 22 could be used to predict results from a specific subject. We expect that additional model 
1 complexity may be necessary to accurately predict subject-specific outputs, even with known or

2 calibrated material properties.

3 Future work will involve creating and evaluating a statistical shape model (SSM) of the

4 lumbar spine and employing the model for probabilistic analysis. One advantage of the method

5 for generating automated lumbar spine FE models is that the geometry can be parameterized

6 through the use of an SSM, something that has not been possible previously (Dreischarf et al.,

7 2014; Sarkalkan et al., 2014). With the SSM and automated methods the primary modes of shape

8 variation in the lumbar spine can be correlated with biomechanical outputs from FE models.

9 Probabilistic methods can also be used to evaluate how variations in shape and soft tissue

10 material properties affect normal biomechanics and how implants work in spines with anatomical

11 shape variations.

12 The current study evaluated lumbar spine FE models created through automated methods.

13 Mesh convergence, semi-direct validation, and indirect validation studies were performed. To the 14 authors' knowledge this is the first study to create and evaluate many full lumbar FE models of 15 different specimens using the same modeling methods. A large range of biomechanical results 16 were produced solely due to geometric variation in the 18 specimens simulated. The studies

17 showed that the automated models can be used to reliably evaluate lumbar spine biomechanics, 18 specifically within our intended context of use: in pure bending modes, under relatively low non19 injurious in vivo simulated loads, to predict torque rotation response, disc pressures, and facet 20 forces.

\section{Acknowledgement}


This work was supported in part by the U.S. National Science Foundation under Grant

846845.

\section{References}

Anderson, A., Ellis, B., Weiss, J., 2007. Verification, validation and sensitivity studies in computational biomechanics. Comput. Methods Biomech. Biomed. Engin. 10, 171-184.

Ayturk, U., 2007. Development and validation of a three dimensional high resolution nonlinear finite element model of an L3/L4 functional spinal unit. M.S. Thesis. Colorado State University.

Ayturk, U.M., Puttlitz, C.M., 2011. Parametric convergence sensitivity and validation of a finite element model of the human lumbar spine. Comput. Methods Biomech. Biomed. Engin. 14, 695-705.

Bischoff, J.E., Dai, Y., Goodlett, C., Davis, B., Bandi, M., 2014. Incorporating Population-Level Variability in Orthopedic Biomechanical Analysis: A Review. J. Biomech. Eng. 136, 021004.

Brinckmann, P., Grootenboer, H., 1991. Change of disc height, radial disc bulge, and intradiscal pressure from discectomy. An in vitro investigation on human lumbar discs. Spine (Phila. Pa. 1976).

Campbell, J.Q., Petrella, A.J., 2015. An Automated Method for Landmark Identification and Finite Element Modeling of the Lumbar Spine. IEEE Trans. Biomed. Eng. 62, 2709-2716.

Cegoñino, J., Moramarco, V., Calvo-Echenique, A., Pappalettere, C., Pérez del Palomar, A., 2014. A Constitutive Model for the Annulus of Human Intervertebral Disc: Implications for Developing a Degeneration Model and Its Influence on Lumbar Spine Functioning. J. Appl. Math.

Charles, Y.P., Lima, L.V.P.C., Persohn, S., Rouch, P., Steib, J.P., Skalli, W., 2013. Influence of an auxiliary facet system on intervertebral discs and adjacent facet joints. Spine J. 13, 12931300 .

Coombs, D., Bushelow, M., Laz, P., Rao, M., Rullkoetter, P., 2013. Stepwise validated finite element model of the human lumbar spine. ASME 2013 Conf. Front. Med. Devices Appl. Comput. Model. Simul. Am. Soc. Mech. Eng.

Dreischarf, M., Zander, T., Shirazi-Adl, A., Puttlitz, C.M., Adam, C.J., Chen, C.S., Goel, V.K., Kiapour, A., Kim, Y.H., Labus, K.M., Little, J.P., Park, W.M., Wang, Y.H., Wilke, H.J., Rohlmann, A., Schmidt, H., 2014. Comparison of eight published static finite element models of the intact lumbar spine: Predictive power of models improves when combined together. J. Biomech. 47, 1757-1766. 
Eberlein, R., Holzapfel, G. a., Fröhlich, M., 2004. Multi-segment FEA of the human lumbar spine including the heterogeneity of the annulus fibrosus. Comput. Mech. 34, 147-163.

Eberlein, R., Holzapfel, G., Schulze-Bauer, C., 2001. An Anisotropic Model for Annulus Tissue and Enhanced Finite Element Analysis of Intact Lumbar Disc Bodies. Comput. Methods Biomech. Biomed. Eng. 4, 209-229.

Erdemir, A., Guess, T.M., Halloran, J., Tadepalli, S.C., Morrison, T.M., 2012. Considerations for reporting finite element analysis studies in biomechanics. J. Biomech. 45, 625-633.

Galloway, F., Kahnt, M., Ramm, H., Worsley, P., Zachow, S., Nair, P., Taylor, M., 2013. A large scale finite element study of a cementless osseointegrated tibial tray. J. Biomech. 46, 19001906.

Guan, Y., Yoganandan, N., Moore, J., Pintar, F.A., Zhang, J., Maiman, D.J., Laud, P., 2007. Moment-rotation responses of the human lumbosacral spinal column. J. Biomech. 40, 1975-1980.

Henninger, H.B., Reese, S.P., Anderson, A.E., Weiss, J.A., 2010. Validation of computational models in biomechanics. Proc. Inst. Mech. Eng. H. 224, 801-812.

Heuer, F., Schmidt, H., Claes, L., Wilke, H., 2007. Stepwise reduction of functional spinal structures increase vertebral translation and intradiscal pressure. J. Biomech.

Holzapfel, G.A., Schulze-Bauer, C.A.J., Feigl, G., Regitnig, P., 2005. Single lamellar mechanics of the human lumbar anulus fibrosus. Biomech. Model. Mechanobiol. 3, 125-140.

Jones, A.C., Wilcox, R.K., 2008. Finite element analysis of the spine: Towards a framework of verification, validation and sensitivity analysis. Med. Eng. Phys. 30, 1287-1304.

Lee, K.K., Teo, E.C., 2005. Material sensitivity study on lumbar motion segment (L2-L3) under sagittal plane loadings using probabilistic method. J. Spinal Disord. Tech. 18, 163-170.

Little, J.P., Adam, C.J., Evans, J.H., Pettet, G.J., Pearcy, M.J., 2007. Nonlinear finite element analysis of anular lesions in the L4/5 intervertebral disc. J. Biomech. 40, 2744-2751.

Malandrino, A., Noailly, J., Lacroix, D., 2013. Regional annulus fibre orientations used as a tool for the calibration of lumbar intervertebral disc finite element models. Comput. Methods Biomech. Biomed. Engin. 16, 923-928.

Moramarco, V., Pérez del Palomar, a., Pappalettere, C., Doblaré, M., 2010. An accurate validation of a computational model of a human lumbosacral segment. J. Biomech. 43, 334342. 
Niemeyer, F., Wilke, H.J., Schmidt, H., 2012. Geometry strongly influences the response of numerical models of the lumbar spine-A probabilistic finite element analysis. J. Biomech. $45,1414-1423$.

Niosi, C.A., Wilson, D.C., Zhu, Q., Keynan, O., Wilson, D.R., Oxland, T.R., 2008. The effect of dynamic posterior stabilization on facet joint contact forces: an in vitro investigation. Spine (Phila. Pa. 1976). 33, 19-26.

Nolte, L., Panjabi, M., Oxland, T., 1990. Biomechanical properties of lumbar spinal ligaments. Clin. Implant Mater. Adv. Biomater. 9, 663-668.

Pearcy, M.J., 1985. Stereo radiography of lumbar spine motion., Acta orthopaedica Scandinavica. Supplementum.

Pearcy, M.J., Portek, I., Shepherd, J., 1984. Three Dimensional Xray Analysis of Normal Movement in the Lumbar Spine. Spine (Phila. Pa. 1976). 9, 294-297.

Pearcy, M.J., Tibrewal, S.B., 1984. Axial rotation and lateral bending in the normal lumbar spine measured by three-dimensional radiography. Spine (Phila. Pa. 1976). 9, 582-587.

Rao, M., 2012. Explicit Finite Element Modeling of the Human Lumbar Spine. Ph.D. Thesis. University of Denver.

Rohlmann, a, Neller, S., Claes, L., Bergmann, G., Wilke, H.J., 2001. Influence of a follower load on intradiscal pressure and intersegmental rotation of the lumbar spine. Spine (Phila. Pa. 1976). 26, E557-E561.

Rohlmann, A., Zander, T., Schmidt, H., Wilke, H.J., Bergmann, G., 2006. Analysis of the influence of disc degeneration on the mechanical behaviour of a lumbar motion segment using the finite element method. J. Biomech. 39, 2484-2490.

Sarkalkan, N., Weinans, H., Zadpoor, A.A., 2014. Statistical shape and appearance models of bones. Bone 60, 129-140.

Sawa, A.G.U., Crawford, N.R., 2008. The use of surface strain data and a neural networks solution method to determine lumbar facet joint loads during in vitro spine testing. J. Biomech. 41, 2647-2653.

Shirazi-Adl, A., 2006. Analysis of large compression loads on lumbar spine in flexion and in torsion using a novel wrapping element. J. Biomech. 39, 267-275.

Tsouknidas, A., Savvakis, S., Asaniotis, Y., Anagnostidis, K., Lontos, A., Michailidis, N., 2013. The effect of kyphoplasty parameters on the dynamic load transfer within the lumbar spine considering the response of a bio-realistic spine segment. Clin. Biomech. 28, 949-955. 
1 Viceconti, M., Olsen, S., Nolte, L.P., Burton, K., 2005. Extracting clinically relevant data from

2 finite element simulations. Clin. Biomech. 20, 451-454.

3 Wilke, H., Neef, P., Hinz, B., Seidel, H., Claes, L., 2001. Intradiscal pressure together with 4 anthropometric data--a data set for the validation of models. Clin. Biomech. (Bristol, Avon) $5 \quad 16$ Suppl 1, S111-S126.

6 Wilson, D.C., Niosi, C.A., Zhu, Q.A., Oxland, T.R., Wilson, D.R., 2006. Accuracy and 7 repeatability of a new method for measuring facet loads in the lumbar spine. J. Biomech. $8 \quad 39,348-353$.

9 
Fig. 1. The three modeling tasks used to support V\&V of the automated lumbar spine FE modeling algorithm.

\section{Table 1}

Material properties used in the mesh convergence and indirect validation simulations. Studies referenced in table 1: a) Cegoñino et al., 2014; b) Dreischarf et al., 2014; c) Little et al., 2007; d) Moramarco et al., 2010; e) Rao, 2012; f) Ayturk and Puttlitz, 2011; g) Nolte et al., 1990; h) Rohlmann et al., 2006; i) Eberlein et al. 2004, 2001; j) Malandrino et al., 2013; k) Holzapfel et al., 2005.

Fig 2. Views of the L1-LS1 FE model in flexion/extension, lateral bending and axial rotation.

Fig 3. Images of the 18 automatically generated full lumbar FE models highlighting diversity of size, shape, and alignment.

Fig 4. Mesh convergence results showing the percent change in output variables rotation, disc pressure, and left/right facet force when the model was subjected to left/right axial rotation, left/right lateral bending, flexion, and extension. Comparisons were made between the original template mesh adopted from Rao (2012) and a second mesh in which element density was doubled.

Fig 5. Semi-direct validation torque rotation curves for L1-S1. Experimental data (Experiment), FE results from the current study (Automated-FE), FE results from Rao (Rao2012).

\section{Table 2}

RMS error results of the semi-direct validation study (in degrees) for L1-S1 and FSU (average RMS error over all five FSUs).

Fig 6. Torque rotation curves for flexion, extension, and right/left lateral bending for all $18 \mathrm{FE}$ models (black) and the experimental results from Guan et al. (2007), mean (solid red), \pm 1 standard deviation (dotted red).

Fig 7. Indirect validation results. Modes: range of motion flexion-extension (FE), lateral bending (LB), axial rotation (AR); one-direction bending flexion (F), extension (E), lateral bending $(\mathrm{L})$, axial rotation $(\mathrm{A})$. Moment $(\mathrm{M})$, Follower Load $(\mathrm{F})$, number of subjects $(\mathrm{N})$, simulation (Sim), experimental (Exp). Authors: a) (Rohlmann et al., 2001), b) (Pearcy and Tibrewal, 1984; Pearcy, 1985; Pearcy et al., 1984), c) (Wilson et al., 2006), d) (Sawa and Crawford, 2008), e) (Niosi et al., 2008), f) (Wilke et al., 2001), g) (Brinckmann and Grootenboer, 1991), h) (Ayturk, 2007; Ayturk and Puttlitz, 2011), i) (Heuer et al., 2007). Simulation data (black) shown with box plots with mean, standard deviation, and all data points. Experimental data (red) with box plots (mean and standard deviation) and/or whisker plots (median and range). 
Mesh Convergence $(n=1)$

L4-L5 Geometry

Generalized Materials

Abaqus/Standard

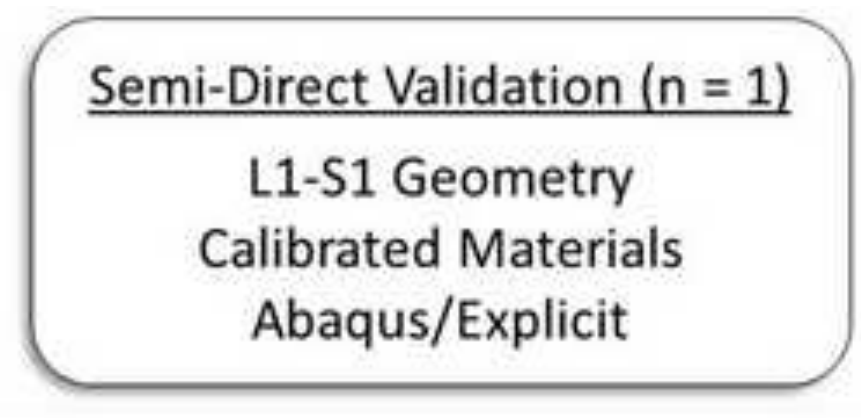

(b)
Indirect Validation $(n=18)$

L1-L5 Geometry

Generalized Materials

Abaqus/Standard

(a)

(c) 


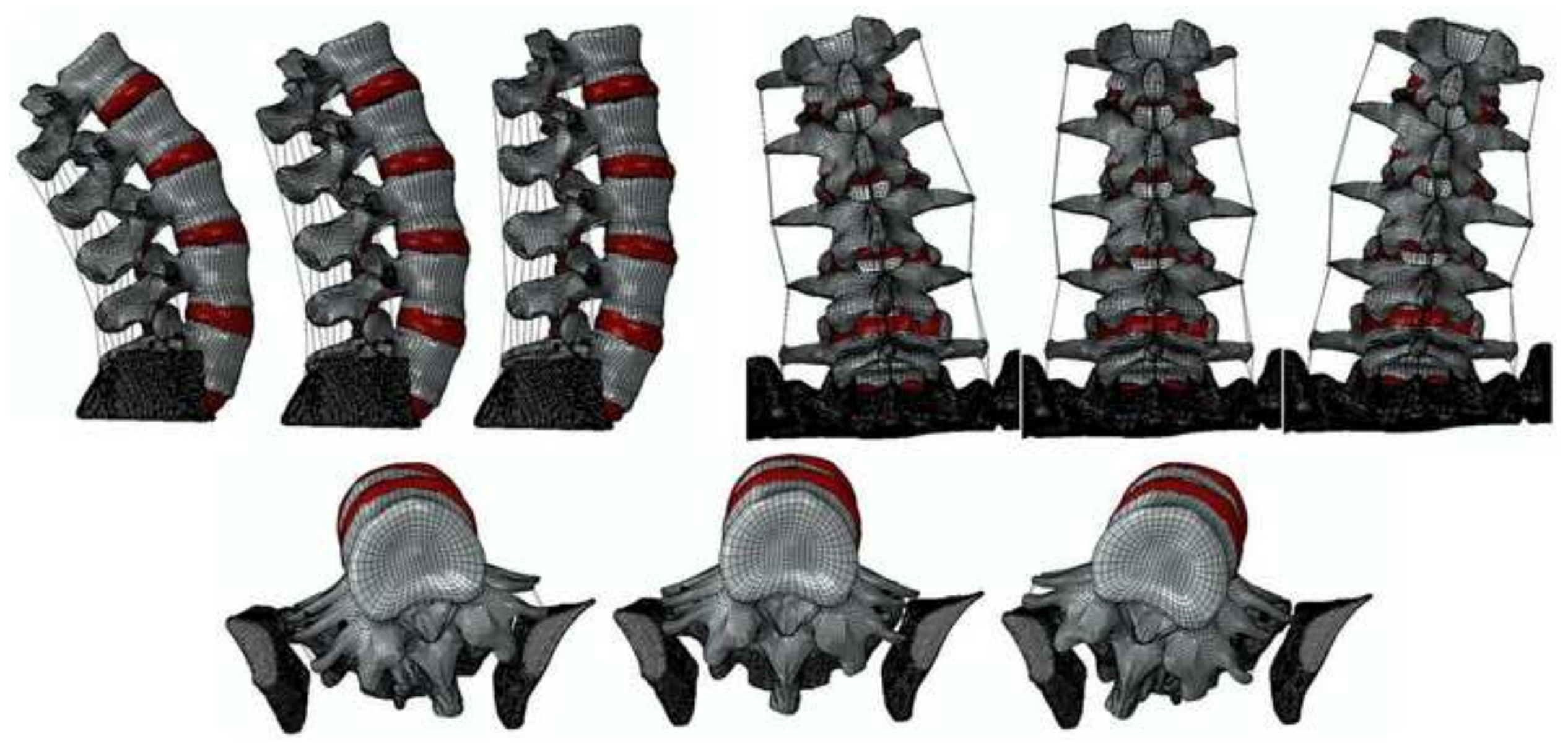



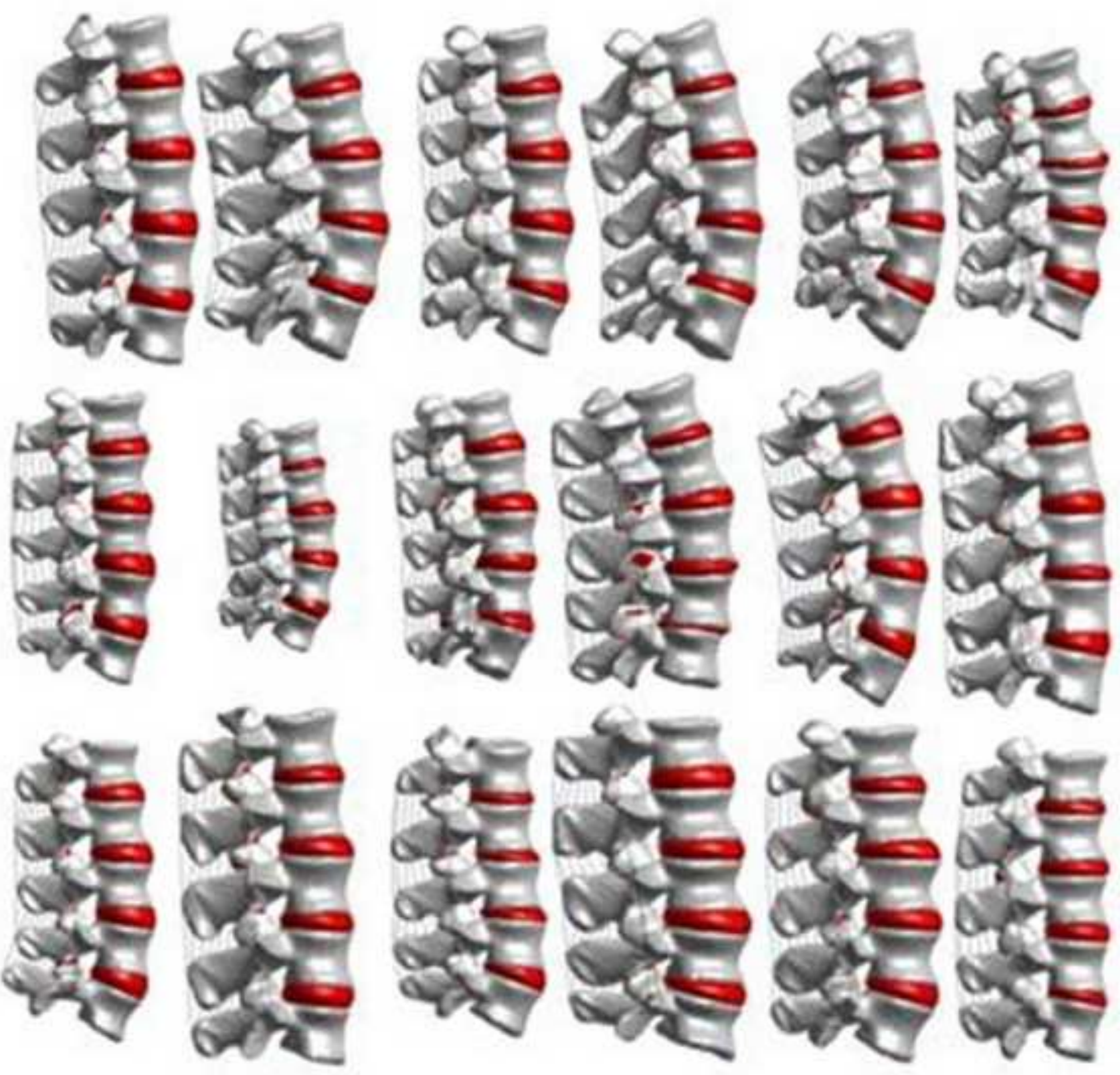


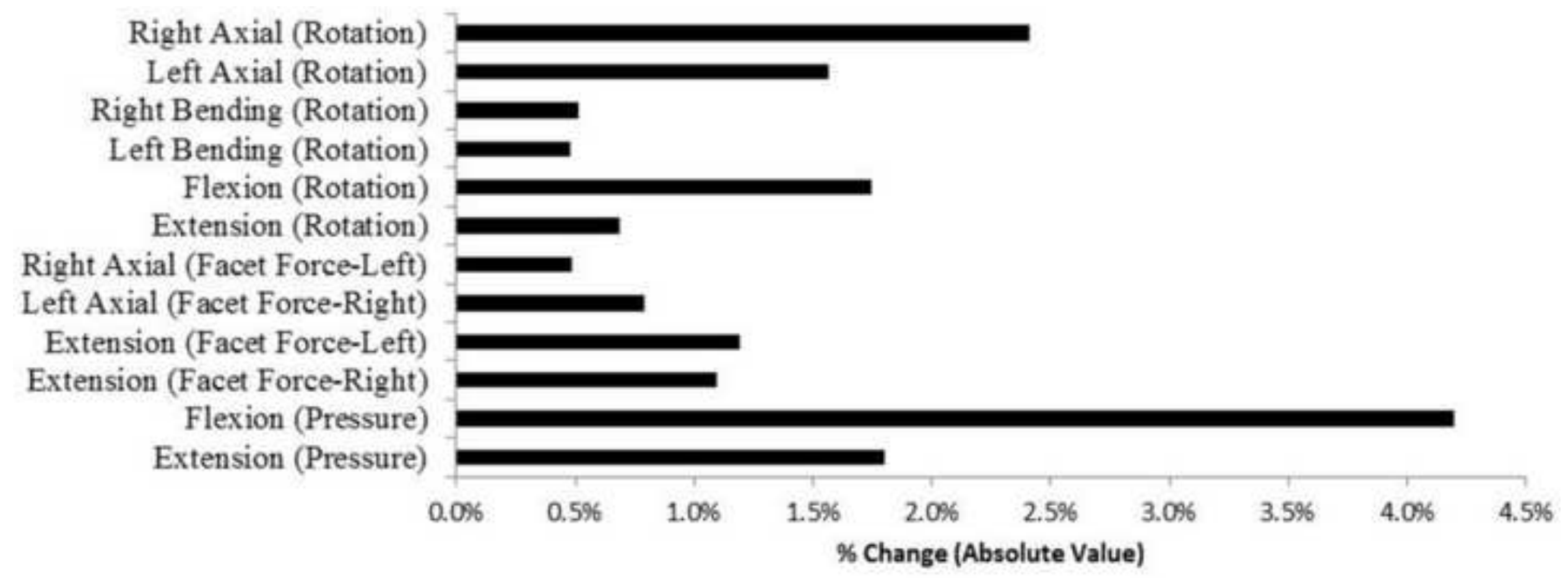



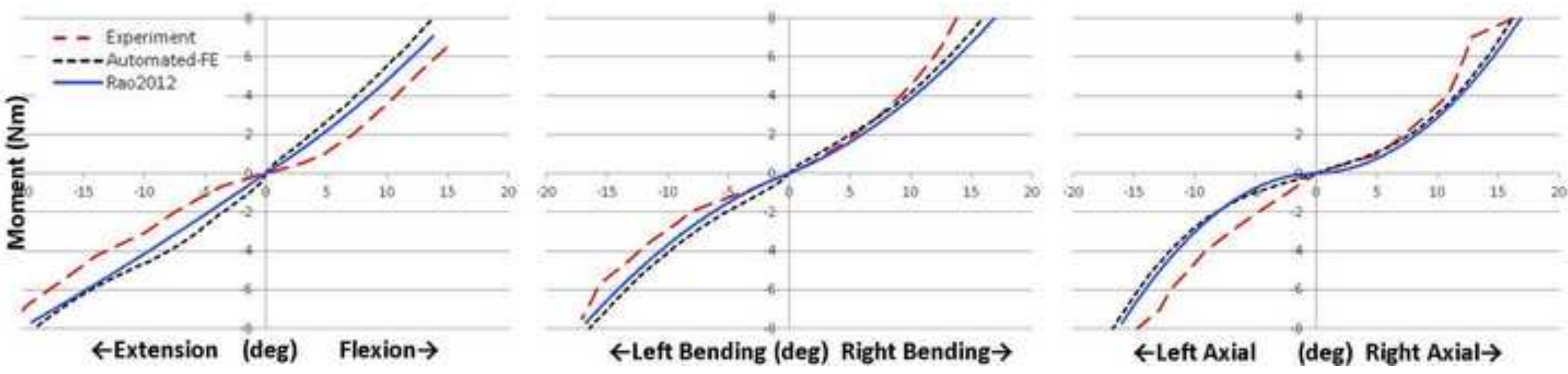

$$
\text { . }
$$




\section{L1-L2}

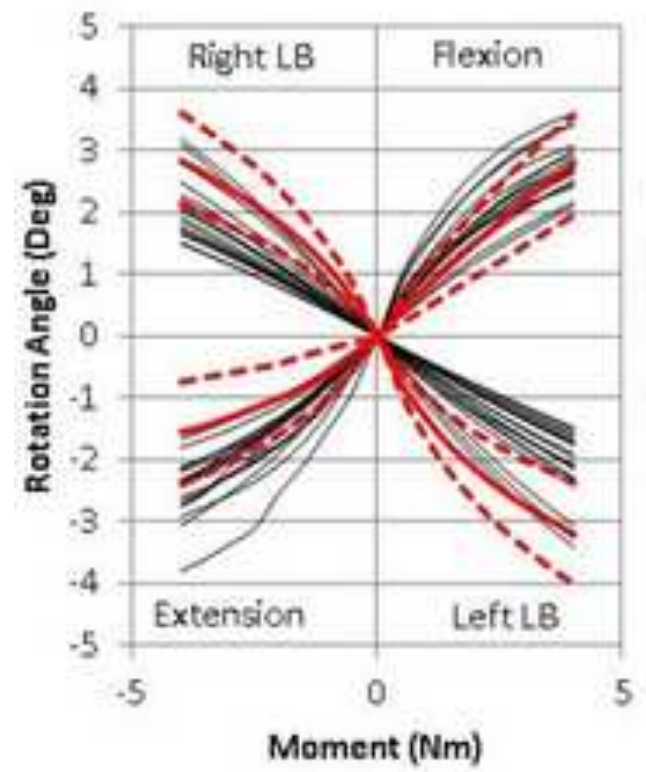

L2-L3

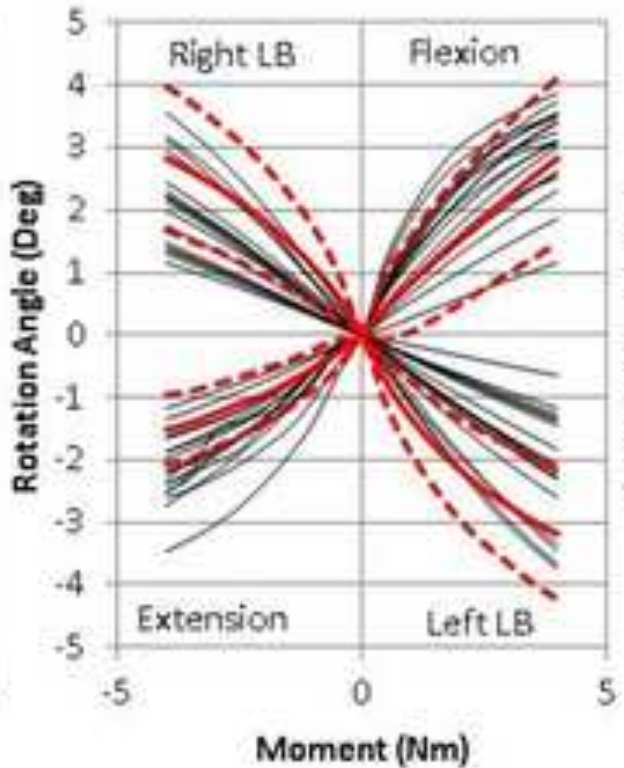

L3-L4

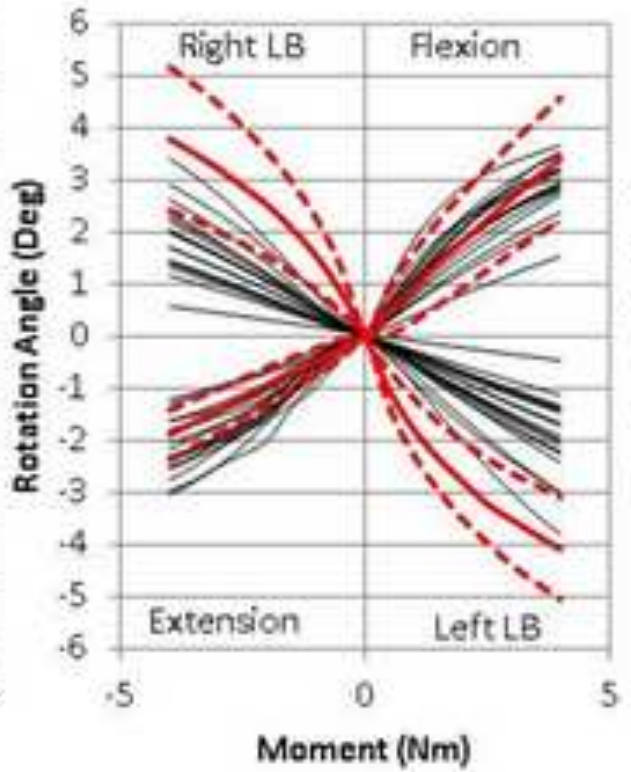

L4-L5

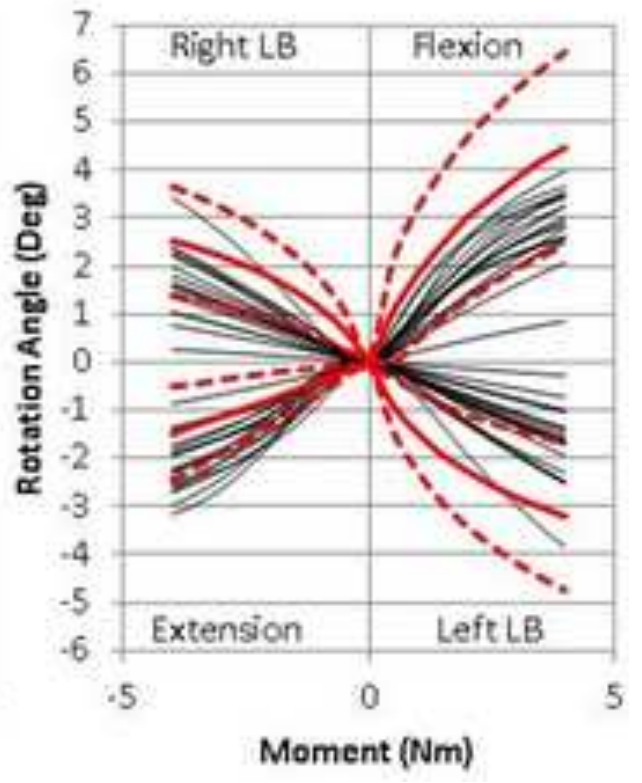




\begin{tabular}{|c|c|c|c|c|c|c|}
\hline fode & Level & $\mathrm{M}(\mathrm{Nm}$ & $\mathrm{F}(\mathrm{N}$ & & $N_{x}$ & Author \\
\hline$F E$ & $1.1-1.5$ & 7.5 & 0 & 17 & 10 & a \\
\hline $\mathrm{IB}$ & $1.1-1.5$ & 75 & 0 & 17 & 10 & a \\
\hline $\mathrm{AR}$ & $\mid 1.55$ & 7.5 & 0 & 16 & 10 & 口 \\
\hline $\mathrm{F}$ & $1.1-5.5$ & 7.5 & 0 & 18 & 10 & $\mathbf{a}$ \\
\hline E & 1.1 .5 & 7.5 & 0 & 17 & 10 & a \\
\hline L. & $\mid 1,1.15$ & 7.5 & 0 & 35 & 10 & a \\
\hline$\Lambda$ & 1.1 .1 .5 & 7.5 & 0 & 34 & 10 & \\
\hline
\end{tabular}

Rotation (deg) o

\begin{tabular}{|c|c|c|c|c|c|c|}
\hline $\mathrm{E}$ & 1.1 .12 & 75 & 500 & 16 & II & b \\
\hline E & $12-13$ & 7.5 & 500 & 16 & 11 & b \\
\hline $\mathrm{E}$ & $1.3-1.4$ & 75 & 500 & 16 & 11 & b \\
\hline E & $1.4-1.5$ & 75 & 500 & 16 & 11 & b \\
\hline I. & 1.1 .12 & 7.8 & 700 & 34 & 10 & b \\
\hline 1. & $1.2-13$ & 7.8 & 700 & 34 & 10 & $b$ \\
\hline L. & $1.3-1.4$ & 7.8 & 700 & 34 & 10 & $\bar{b}$ \\
\hline L & $1.4-15$ & 7.8 & 700 & 34 & 10 & $\mathrm{~b}$ \\
\hline A & 1.1 .1 .2 & 5.5 & 720 & 33 & 10 & $\bar{b}$ \\
\hline $\mathrm{A}$ & 1.2 .1 .3 & 5,5 & 720 & 33 & 10 & $b$ \\
\hline$\Lambda$ & 1.3-1.A & 5.5 & 720 & 33 & 10 & $\bar{b}$ \\
\hline A & L.A-L.S & 55 & 720 & 33 & 10 & $\bar{b}$ \\
\hline
\end{tabular}

Rotation (deg) 4

\begin{tabular}{|c|c|c|c|c|c|c|}
\hline F & $1.1-1.2$ & 75 & 0 & 36 & 12 & $c$ \\
\hline E & 1.1 .12 & 7.5 & 0 & 34 & 12 & $c$ \\
\hline 1. & $1,1=1,2$ & 7.5 & 0 & 35 & 12 & c \\
\hline A & L.I.L.2 & 75 & 0 & 34 & 12 & $\mathrm{c}$ \\
\hline A & $1.3 . \mathrm{A}$ & 7.5 & 0 & 34 & 8 & d \\
\hline A & L.3.LA & 7.5 & 0 & 34 & 20 & $c$ \\
\hline $\mathrm{E}$ & $1.3-1 \mathrm{~A}$ & 7,5 & 0 & 34 & 8 & $d$ \\
\hline $\mathrm{E}$ & $L .3-1 \mathrm{~A}$ & 7.5 & 0 & 34 & 20 & $\mathrm{c}$ \\
\hline $\mathrm{F}$ & $53-1 A$ & 75 & 0 & 36 & 20 & $\mathrm{c}$ \\
\hline L. & L.3-1A & 7.5 & 0 & 35 & 20 & $\mathrm{c}$ \\
\hline
\end{tabular}

Facet Foree (N) 0

\begin{tabular}{|c|c|c|c|c|c|c|}
\hline E. & L.4.LS & 7.5 & 500 & 16 & $\mathrm{I}$ & $\mathrm{f}$ \\
\hline 1. & $1.4-1.5$ & 7.8 & 700 & 34 & 1 & $\mathrm{f}$ \\
\hline A & $L-4.1 .5$ & 5,5 & 720 & 33 & 1 & $\mathrm{f}$ \\
\hline C & $1.2 \cdot 1.3$ & 0 & 300 & 18 & 5 & $g$ \\
\hline$C$ & 1.2 .1 .3 & 0 & 1000 & 18 & 5 & $B$ \\
\hline C & $1.3-1 \mathrm{~A}$ & 0 & 300 & 18 & 1 & $\mathrm{~g}$ \\
\hline C & $1.3 .1 \mathrm{~A}$ & 0 & 1000 & 18 & 1 & $g$ \\
\hline$C$ & LA-1.S & 0 & 300 & 18 & 5 & $\mathrm{~g}$ \\
\hline c & LA-LS & 0 & 1000 & 18 & 5 & 8 \\
\hline$F$ & L.2-1.3 & 3 & 0 & 18 & 6 & h \\
\hline$F$ & $1.3-1 . A$ & 3 & 0 & 18 & 6 & $\bar{b}$ \\
\hline $\mathrm{F}$ & $L A-L .5$ & 1 & 0 & 18 & 8 & i \\
\hline$F$ & $L A-L S$ & 2.3 & 0 & 18 & 8 & $i$ \\
\hline$F$ & LA-LS & 5 & 0 & 18 & 8 & $i$ \\
\hline$F$ & LA-LS & 75 & 0 & 18 & 8 & i \\
\hline$E$ & $1.2-1.3$ & 3 & 0 & 18 & 6 & b \\
\hline E & $13-1.4$ & 3 & 0 & 18 & 6 & b \\
\hline E & 1.4 .1 .5 & 1 & 0 & 18 & 8 & $i$ \\
\hline $\mathrm{E}$ & $1.4-15$ & 2.5 & 0 & 18 & 8 & $i$ \\
\hline E & L.4.L5 & 5 & 0 & 18 & 8 & i \\
\hline $\mathrm{E}$ & LA-I.5 & 7.5 & 0 & 17 & 8 & $i$ \\
\hline
\end{tabular}

Disc Pressure (MPa) -0.5

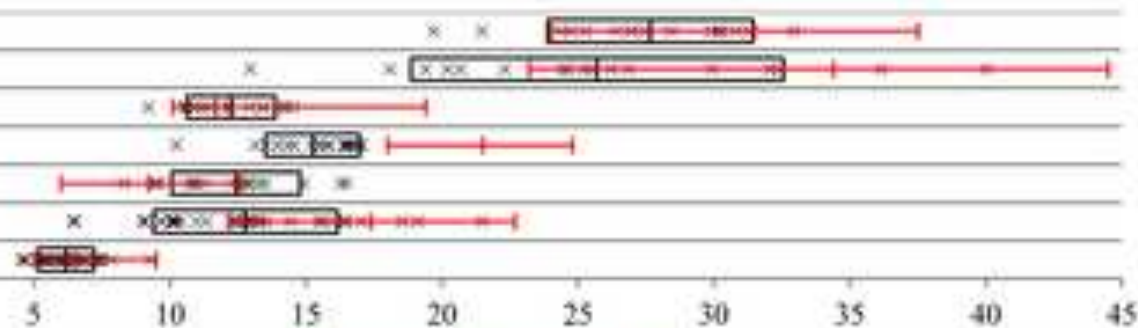

$x)=1$
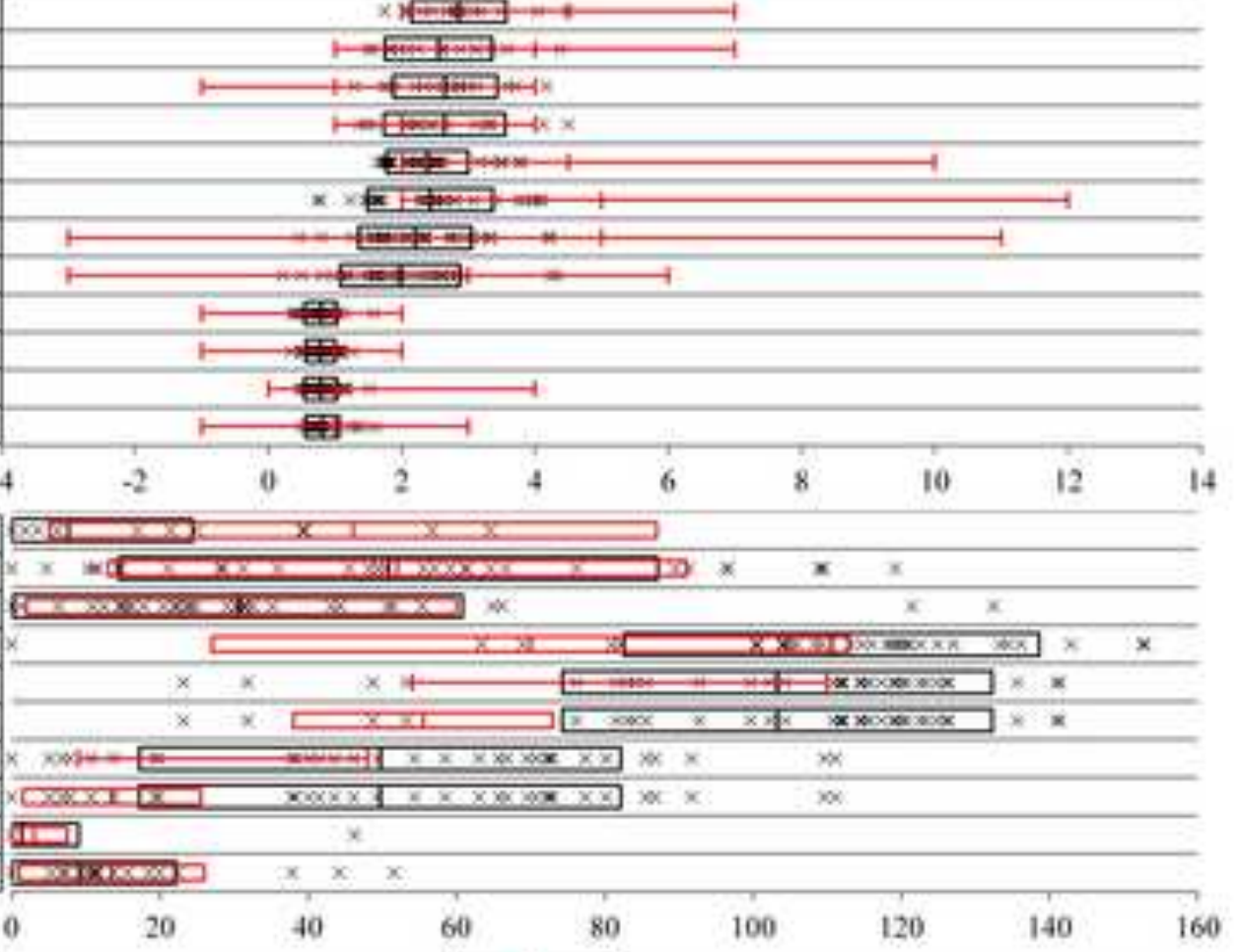

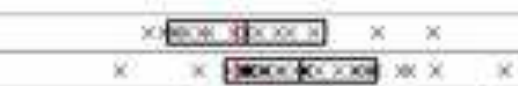

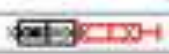

$x \sqrt{x+1 \times=3} \times x$

क्यक।

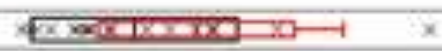

$\times x \times x$

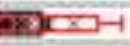

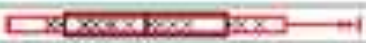

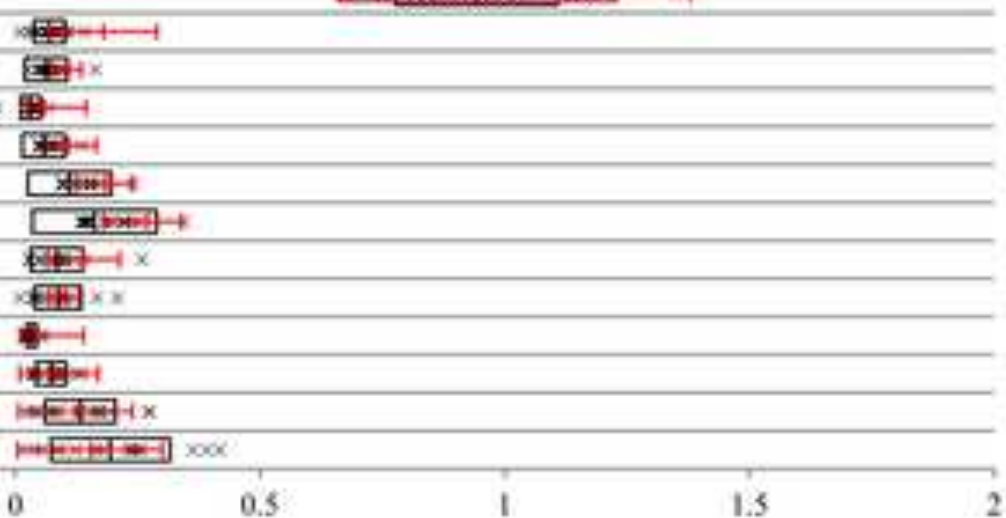




\section{Table1}

\begin{tabular}{|c|c|c|c|c|c|c|}
\hline Bones / Endplates & \multicolumn{6}{|l|}{$\operatorname{Rigid}^{\mathrm{a}, \mathrm{b}, \mathrm{c}, \mathrm{d}, \mathrm{e}}$} \\
\hline Ligaments & \multicolumn{6}{|c|}{ Tension Only, Exponential Force-Displacement ${ }^{\mathrm{f} g, \mathrm{~h}}$} \\
\hline \multirow{5}{*}{ Annulus Fibrosis } & \multicolumn{2}{|c|}{ Matrix } & Fibers & Anterior & Lateral & Posterior \\
\hline & \multirow{2}{*}{$\mathrm{C} 10$ (MPa) } & \multirow{2}{*}{$0.25^{i}$} & k1 (MPa) & $2^{j}$ & $3^{i}$ & $5^{j}$ \\
\hline & & & k2 & $190^{j}$ & $45^{i}$ & $10^{\mathrm{j}}$ \\
\hline & \multirow{2}{*}{$\mathrm{D}(1 / \mathrm{MPa})$} & \multirow{2}{*}{$0^{i}$} & Angle (deg) & $26^{k}$ & $34.9^{k}$ & $43^{k}$ \\
\hline & & & Kappa & 0 & 0 & 0 \\
\hline Nucleus Pulposus & \multicolumn{6}{|c|}{ Fluid-Cavity $\mathrm{K}=2200 \mathrm{MPa}^{\mathrm{e}}$} \\
\hline Facet Cartilage & \multicolumn{6}{|c|}{ Frictionless, Softened Contact, Linear Pressure-Overclosure, k=100 MPa/mm } \\
\hline
\end{tabular}




\begin{tabular}{|c|c|c|c|c|}
\hline \multicolumn{2}{|c|}{ RMS Error } \\
\hline Automated FE & \multicolumn{2}{|c|}{ vs Experiment } & \multicolumn{2}{c|}{ vs FE Rao 2012 } \\
\hline & FSU & L1-S1 & FSU & L1-S1 \\
\hline Flexion-Extension & 0.6 & 3.3 & 0.2 & 1.1 \\
\hline Lateral Bending & 0.3 & 1.7 & 0.2 & 0.8 \\
\hline Axial Rotation & 0.3 & 2.1 & 0.1 & 0.6 \\
\hline
\end{tabular}

